\title{
Emerging permanent filler technologies: focus on Aquamid
}

\author{
Paul S Yamauchi ${ }^{1,2}$ \\ 'Dermatology Institute and Skin \\ Care Center, Santa Monica, CA, USA; \\ ${ }^{2}$ Division of Dermatology, David \\ Geffen School of Medicine at UCLA, \\ Los Angeles, CA, USA
}

This article was published in the following Dove Press journal:

Clinical, Cosmetic and Investigational Dermatology

10 October 2014

Number of times this article has been viewed
Correspondence: Paul S Yamauchi Dermatology Institute and Skin Care Center, 200I Santa Monica Blvd, Ste II 60W, Santa Monica, CA 90404, USA $\mathrm{Tel}+\mathrm{I} 3108294104$

Fax +I 310 829 4I50

Email paulyamauchi@yahoo.com

\begin{abstract}
A plethora of soft tissue fillers have been developed within the past decade to correct the cutaneous changes that occur with photoaging. Such fillers, whether nonpermanent, semipermanent, or permanent, are widely used to fill undesired facial rhytides. In addition, fillers are employed to correct atrophy of the face as well as other parts of the body such as the dorsum of the hands through volumization and contouring. The extensive long-term safety outcomes reported with fillers and the ease with which they are administered make them an ideal choice to correct rhytides and to contour the face. However, as with any cosmetic procedure, in order to ensure high patient satisfaction and a safe outcome, proper training in injection techniques, the choice of the proper candidate, and awareness of potential adverse events are essential. This review article focuses on the permanent filler, Aquamid, which is composed of polyacrylamide hydrogel.
\end{abstract}

Keywords: Aquamid, rejuvenation, skin aging, soft tissue fillers, polyacrylamide

\section{Introduction}

Injectable fillers have become one of the mainstay treatments for nonsurgical treatment of wrinkles and facial contouring. A plethora of fillers are currently available and even more so internationally. Because of unique characteristics of each kind of filler, there are different indications as well as advantages and disadvantages of each filler type. How these fillers are distinguished from one another depends on the active ingredient used, the degree of viscosity, the source of the material, and the indications for use. Proper selection type of the filler for the appropriate areas to be injected is paramount to avoid potential complications and to achieve the desired outcome.

Fillers are divided into three classes: nonpermanent, semipermanent, and permanent. Nonpermanent fillers are the most commonly used. Some common materials that make up nonpermanent fillers include hyaluronic acid and collagen. These are of short duration with typical lengths of several months to 1 year and are eventually reabsorbed through macrophage activation. Semipermanent fillers have a longer duration of placement with time periods of years and typically result in a foreign body reaction that elicits fibroblast activation and collagenesis at the site of the material placed in the dermis. Calcium hydroxyapatite and poly-L-lactic are examples of semipermanent fillers. Permanent fillers are the longest acting and also involve fibrogenesis and collagen production. Such fillers include materials composed of silicone, polymethylmethacrylate, and polyacrylamide hydrogel (Aquamid). submit your manuscript | www.dovepress.com

http://dx.doi.org//0.2147/CCID.S46650
Clinical, Cosmetic and Investigational Dermatology 20I4:7 26I-266

(c) (i) (5) 2014 Yamauchi. This work is published by Dove Medical Press Limited, and licensed under Creative Commons Attribution - Non Commercial (unported, v3.0) License. The full terms of the License are available at http://creativecommons.org/licenses/by-nc/3.0/. Non-commercial uses of the work are permitted without any further permission from Dove Medical Press Limited, provided the work is properly attributed. Permissions beyond the scope of the License are administered by Dove Medical Press Limited. Information on
how to request permission may be found at: http://www.dovepress.com/permissions.php 


\section{Characteristics of Aquamid and injection techniques}

Aquamid has been used extensively for soft tissue augmentation and body contouring for the past 20 years. ${ }^{1}$ Aquamid was initially developed and marketed by Contura International A/S in Denmark before being acquired in 2013 by Speciality European Pharma Limited (London, UK). Aquamid is a biocompatible and nonabsorbable hydrogel consisting of $97.5 \%$ water and $2.5 \%$ cross-linked polyacrylamide. The gel is manufactured through polymerization of the acrylamide monomers and N,N'-methylenbisacrylamide. ${ }^{1}$ The colorless transparent gel is provided in sterile $1 \mathrm{cc}$ Luer lock syringes. Aquamid is currently approved in several countries in Europe, Asia, the Middle East, and Latin America but is not available in the US.

Aquamid is administered into the subcutaneous tissue with a 27 -gauge needle. The areas to be injected should be thoroughly prepped and cleansed with preparations such as isopropyl alcohol and/or antibacterial cleansers. Injections into the dermal layer should be avoided to prevent the formation of undesired nodules and papules. Local anesthesia either through topical anesthetic creams containing different combinations and strengths of lidocaine, prilocaine, tetracaine, or benzocaine; regional nerve blocks or infiltration; vibratory anesthesia; or a combination of the any of the aforementioned is highly recommended to minimize discomfort to the patient.

The retrograde injection technique is commonly used to administer Aquamid. The body of the needle is inserted entirely into the subcutaneous tissue at a $30^{\circ}$ angle, and the filler is injected with gentle pressure while slowly withdrawing the needle. Care is taken to stop injecting just as the bevel of the needle reaches the dermis to avoid formation of an unwanted papule. Gentle molding can be performed afterward with the gloved injectors' fingers to evenly distribute the filler. The use of a lubricant such as ultrasound gel facilitates molding.

Cross-hatching and fanning are useful techniques for covering a wide area such as the cheeks for lipodystrophy and where a large amount of Aquamid is required. Cross-hatching and fanning are used primarily for volumetric enhancement and facial contouring. A series of retrograde injections are performed into the deep dermal or subdermal layer. This is followed by another series of retrograde injections in a direction perpendicular to the initial set of injections.

The deep depot technique for injecting Aquamid is another method for facial contouring and volumetric enhancement. For example, to augment the malar eminences, the 27-gauge needle is deeply inserted perpendicular into the skin over the zygoma until the tip touches the periosteum. A droplet of Aquamid is injected over the periosteum of the zygoma until noticeable visible enhancement is seen. A series of injections are performed over the zygoma followed by gentle molding.

Histologic analysis of Aquamid injected into the subcutaneous layer revealed bioactive product that underwent cell infiltration and integration into tissues between weeks 1 and 8. ${ }^{2}$ This was in contrast to a comparative filler that contained hyaluronic acid in which there was minimal cell infiltration with the product that remained surrounded uniformly by a thin capsule. However, another study demonstrated that Aquamid dissipated slower than other comparative fillers and remained intact through the formation of fine fibrous capsules at month $9 .{ }^{3}$

Aquamid may be injected to correct deeper rhytides such as deep nasolabial folds and the marionette lines at the depressed corners of the mouth. It can also be utilized for lip augmentation and contouring of the chin, cheeks, and nose. In areas where the subcutaneous layer is thicker, filling with Aquamid generally starts with deeper injections to augment the atrophy of the tissue. Final corrections and adjustments are then accomplished through more superficial injections in smaller volumes below the subdermal junction.

The patient should be seated comfortably on the examination chair in an upright to semi-upright position to allow for normal downward sagging of the face to occur with gravity. Critical features of the folds will be more evident in a more upright position. If the patient is placed supine on the table, then essential details of the face, particularly in the midface, will not be as clearly evident as they will be partially lost due to backward forces. Therefore, the prone position is not recommended for injected fillers into the face. Good light is essential, and sometimes folds may become more prominent and easier to visualize if the light is adjusted at an angle to cast shadows on the wrinkles.

Prior to injecting Aquamid, critical examination of the areas to be injected as well as pre-procedural photographs are highly recommended. Marking the injection sites with an easy to remove marker pen or pencil may be beneficial. The placement of ice or cold compresses before, during, and after the injections can be utilized to provide comfort to the patient and to induce partial vasoconstriction locally and minimize purpura. Following injections, gentle molding and massaging may be performed to evenly distribute the filling agent beneath the skin. Immediate post-procedural photographs are also recommended. The patient should be 
told not to excessively touch the injected areas and minimize exaggerated facial expression for the next 24 hours. Transient swelling and bruising should be warned to the patient beforehand and are natural to occur and frequently inevitable. The occurrence of any unwanted bumps or unevenness is a risk of any filler agent and is usually due to faulty technique. The patient should call and follow-up in the office should this occur, and corrective actions should be exercised.

\section{Clinical efficacy of Aquamid}

The pilot study published in 2003 using Aquamid for esthetic correction was based on 59 subjects. ${ }^{4}$ The most frequent procedure was lip augmentation (72\%) whereby the majority of patients fell into two age groups: 20-25 years and 50-60 years. Cheekbone enhancement was performed in 13\% of the cases. The rest of the patients had correction performed on nasolabial folds, glabella lines, and chin folds. There was almost $100 \%$ patient satisfaction with the esthetic results.

A prospective, multicenter study was conducted looking at the efficacy and safety in 251 subjects after injection of Aquamid for the enhancement of facial deficiencies. ${ }^{5}$ A 1-year follow-up was assessed in all patients that continued in the study. Patients were eligible if they had irregularities and deformities that were caused by acne, aging, surgical procedures, and trauma. Following the injections, 228 subjects were followed for up to 1 year. Results that were assessed to be good or very good by investigators, or felt to be satisfactory or very satisfactory by the subjects were seen in $93 \%$ of the cases. The most common adverse events recorded in 37 cases were erythema, bruising, swelling, itching, and pain. Some rare side effects that were seen were a slight color change at the site of injection and one case of neutropenia. There were no other laboratory abnormalities. There were no severe events that required hospitalization. Over the 1-year period, the efficacy of soft tissue enhancement was maintained. The investigators attributed tissue reactions to the technique of the injection rather than the polyacrylamide hydrogel found in Aquamid. There was no permanent immunologic reaction seen during the first year.

In a follow-up to the previous study, $41 \%$ of the enrollees participated in a follow-observational study 24 months after the first injection. ${ }^{6}$ The nasolabial folds, glabella, and lips were the most common sites for injection. The volume of Aquamid injected varied from $0.2 \mathrm{~mL}$ to $12 \mathrm{~mL}$. The results were deemed in the opinion of the investigators to be good or very good in the 93 patients who completed the 2-year study. Patient-reported outcomes showed that 86 patients felt the degree of improvement to be satisfactory or very satisfactory. There were no serious adverse events seen during the 24-month follow-up period. Several subjects experienced local reactions that were transient with spontaneous resolution. In one situation, a burning sensation persisted for 24 months in the lips after augmentation. In more than $90 \%$ of patients, satisfactory results were obtained with Aquamid, and there were no substantial changes in improvement at the 12- and 24-month follow-up periods.

In the same prospective study, safety and efficacy assessments were measured 36-60 months after the first injection of Aquamid. ${ }^{7,8}$ An average of $4.3 \mathrm{~mL}$ of Aquamid was injected per patient with an average number of sessions of 2.4. The most common areas that were injected were the nasolabial folds (37\%) and the lips (28\%). Investigator global assessment rated the outcome as good or very good in $96.5 \%$ of the patients. Parent-reported outcomes rated a good or very good improvement in $96.5 \%$ of the patients. There were 53 treatment-related adverse events during the study and two serious adverse events. During the entire study period, a total of 53 adverse events and two serious adverse events were classified as treatment related. There were four cases of infections reported and some incidences of skin induration caused by the polyacrylamide hydrogel. All had resolved by the end of the study. Overall, the esthetic outcome in this study was favorable with few side effects occurring.

A US press release in 2009 discussed results of a randomized, double-blinded comparator multicentered study that compared the effectives and safety of Restylane vs Aquamid that involved 316 patients at 13 US centers across the US for the correction of nasolabial folds. Subjects were randomized to receive either Restylane or Aquamid in an $1: 2$ ratio. Up to two touch-up injections were allowed in the study. Through a 1-year course after treatment, the study demonstrated that the both Aquamid and Restylane were well tolerated and safe. The Wrinkle Assessment Scale was used to measure improvement and demonstrated that both Aquamid and Restylane were equally effective 6 months after injections. A 12-month follow-up period showed that Aquamid still maintained correction and that more subjects rated the improvement with Aquamid as improved than with Restylane. The most commonly reported adverse events in both arms of the study that were treatment related during the first 1 year were purpura, swelling, erythema, and pain. Treatment-related adverse events were considered mild and transient with the majority of events resolving in 3 days. Adverse events occurred in $85 \%$ of the patients in both the Aquamid- and Restylane-treated groups. In a 12-24-month follow-up period, only one serious treatment-related adverse 
event occurred that resolved spontaneously, and there were no significant safety concerns. To date, the results of the US study have not been published.

The volumization of the malar area with Aquamid was evaluated in 1,306 patients in a retrospective study with follow-ups ranging from 3 months to 6 years. ${ }^{9}$ A total of 1,241 patients $(95 \%)$ were satisfied initially. Sixty-five patients (5\%) were not satisfied with the outcome due to insufficient injection volumes. The most common complications were unevenness, nodule formation, pain, and displacement of the gel that occurred in $8.4 \%$ of the subjects (111 patients).

A multitude of studies have been conducted to investigate the efficacy of Aquamid to correct facial lipoatrophy in HIV patients. The aim of one noninferiority study was to demonstrate the efficacy of Aquamid vs polylactic acid for the treatment of facial lipoatrophy in HIV-positive adults in a 96-week study. ${ }^{10}$ The Visual Analogue Scale (VAS) was used to measure the primary endpoint, which was patient satisfaction at week 48. Secondary endpoints included the thickness of the cheek and skin, cheek thickness grading of the degree of lipoatrophy, and quality of life assessment. A total of 148 patients were enrolled in the study. The median age was 47 years, $93 \%$ of the participants were men, the median CD4 count was 528 cells $/ \mu \mathrm{L}$, and the median duration of antiretroviral treatment was 12 years. The mean VAS baseline score was 2.8 in both cohorts that increased to 7.5 and 7.1 in the Aquamid and polylactic acid groups, respectively, at week 48. At week 96, the VAS scores were sustained (7.9 and 6.7 in the Aquamid and polylactic acid cohorts, respectively). Likewise, skin and cheek thicknesses and lipoatrophy improvement were similar in both arms. Improvement in the quality of life based on the questionnaires that the subjects answered either remained unchanged or improved. Subcutaneous nodules were observed in $26(37 \%)$ and $28(41 \%)$ patients in the Aquamid and polylactic acid cohorts, respectively. There were four patients in the Aquamid group who developed severe inflammatory nodules after the last injection with a median time of 17 months. The investigators concluded that Aquamid and polylactic acid have similar efficacies in the treatment of HIV facial lipoatrophy, but that Aquamid may be associated with the appearance of more delayed inflammatory nodules.

A smaller study of 32 HIV subjects with facial lipoatrophy demonstrated that larger volumes of Aquamid (8-12 cc) were not associated with a higher rate of complications such as foreign body reactions and infections. ${ }^{11}$ Facial contouring was attained at the end of the study with an improvement in the quality of life. However, 13 of the 18 subjects developed small, palpable nonvisible nodules at the end of the follow-up.

A multicenter, open-label noncomparative study evaluated the efficacy and safety of intradermal facial injections of polyacrylamide hydrogel in HIV-infected patients with severe facial lipoatrophy. ${ }^{12}$ Two to six injections were performed every 4 weeks with Aquamid. Facial ultrasonography and photography were performed at baseline and months 6,12 , and 24 to evaluate efficacy of Aquamid. In addition, patientreported outcomes, quality of life measurements, and adverse events were recorded. A total of 111 patients participated and received at least one injection of Aquamid. The mean cheek skin thickness was $9.7 \mathrm{~mm}$ at baseline, which increased by an average of $4.4 \mathrm{~mm}$ at year 1 and a further $0.87 \mathrm{~mm}$ at year 2 . Based on patient-reported outcomes, independent grading of facial photographs, and physician-reported outcomes, the overall treatment satisfaction scale demonstrated an improvement in HIV lipoatrophy in more than $88 \%$ of patients during all visits. The lipodystrophy-specific ABCD scale showed that quality of life improved significantly over time and correlated with the degree of correction. No severe adverse effects due to Aquamid injections were observed. In general, Aquamid injections were well tolerated and significantly improved the facial lipoatrophy in HIV-positive patients with a correlating improvement in the quality of life.

A prospective study assessed 23 HIV-positive patients with facial lipoatrophy who were randomized to undergo treatment with autologous fat transfer or Aquamid. ${ }^{13}$ Follow-up visits were scheduled at 3, 6, and 12 months after initial evaluation to evaluate clinical efficacy. The primary endpoint in the study used the Global Aesthetic Improvement Scale, which was measured by the investigator 1 year after baseline. Secondary endpoints used the same scale 6 months after baseline. There were no infections or other complications observed in the study. The primary and secondary endpoints demonstrated that autologous fat transfer yielded significantly higher ratings than Aquamid after baseline. The authors concluded autologous fat transfer yielded better outcomes than Aquamid in correcting facial lipodystrophy due to deeper filling with autologous fat transfer.

The safety and efficacy of Aquamid was evaluated in HIV-positive patients with facial lipodystrophy by receiving different volumes of Aquamid at different time intervals. ${ }^{14}$ A total of 31 patients were randomized to receive Aquamid in two cohorts: A and B. In cohort A, the lipoatrophy was corrected by injecting up to $8 \mathrm{~mL}$ of Aquamid in the first session. Patients were then retreated every 8th week with touch-up injections until full correction was observed. In cohort B, 
lipoatrophy was treated by injecting $2 \mathrm{~mL}$ of Aquamid each session. The subjects were injected every 8 weeks until full correction was observed. The subjects in cohort A noted a greater improvement after receiving the initial $8 \mathrm{~mL}$ bolus of Aquamid. On average, patients in cohort B noted improvement of their face after receiving their fourth injection session. There were no safety differences between the two cohorts, and there were no cases of foreign body reaction, infection, or displacement of the polyacrylamide hydrogel.

\section{Safety of Aquamid}

As with any filler agent, whether it is nonpermanent, semipermanent, or permanent, the benefits and the potential risks must be discussed with the patient. For the most part, true complications are not common with soft tissue fillers if proper injection technique is performed and the appropriate patient is selected. Unevenness, ridging, papules, and nodule formation can occur with any filler agent and arise mainly due to faulty and improper injection technique.

One study evaluated 98 patients who attained unsatisfactory results and complications from injections with Aquamid. ${ }^{15}$ The most common adverse events seen were inflammation (52\%), asymmetry (32\%), irregularity (18\%), abscess formation and infection (11\%), and product displacement $(8 \%)$. A severe anaphylaxis reaction was seen in one subject 1 week after injection that led to significant complications. Histologic analysis revealed granuloma formation (17\%), fibrosis (17\%), and fat necrosis (9\%). Visible Aquamid-related complications were corrected after physical extraction of the injected polyacrylamide hydrogel. However, hyperpigmentation and skin necrosis remained unchanged during the study. In eight subjects, the Aquamid product could be extracted by drainage and irrigation. For eight patients, the polyacrylamide hydrogel could not be extracted by squeezing and irrigation entirely. Three patients experienced relapse of product reaccumulation after complete extraction was performed.

A retrospective study in a follow-up to 542 patients who received facial injections of Aquamid showed 7.7\% of patients (42) developed complications such as edema, unevenness and lumpiness, abscess formation, alteration in facial appearance, migration of product, and pain. ${ }^{16}$

Delayed immune-mediated adverse effects due to Aquamid injections were assessed in a prospective study of 10 patients. ${ }^{17}$ The average latency period before the onset of complications was 10 months (range 2-36). Tender, inflamed nodules and pseudo-abscesses were observed. Tender, inflammatory nodules - granulomas - with pseudo-abscesses were evident. After an average follow-up time of 20.1 months, five patients were in remission, two developed recurrent nodules, and the remaining three were lost to follow-up with one of them in remission.

Twenty-four patients underwent surgical extraction following complications after facial injections with Aquamid over a 6-year period. ${ }^{18}$ Some of the adverse events that were observed included infection, nodule formation, hematoma, and displacement of product. Ultimately, one patient underwent drainage to extract with product and the other 23 patients underwent surgical extraction of the Aquamid.

A case-control study was conducted to compare 30 cytology specimens and 77 biopsies from 59 subjects who developed adverse reactions to Aquamid, and two cytology specimens and 54 biopsies from 28 control patients without complications. ${ }^{19}$ Samples from five patients and four controls could not be assessed due to contamination from bacterial pathogens. The remaining specimens from the 54 patients and 24 control subjects were analyzed for Gram staining and bacterial culture, 16S rRNA gene sequencing, and fluorescence in situ hybridization. Propionibacterium acnes and Staphylococcus epidermidis, which are normal skin flora, were cultured from 53 patients (98\%), and none from the control group. Five years after injection, the bacteria were still detected in areas of complications. The study demonstrated that commensal bacteria of the skin can produce long-term infections in polyacrylamide hydrogel due to the formation of bacterial biofilm. To circumvent bacterial infection and biofilm development, sterile injection techniques and thorough skin cleansing are essential. In addition, prophylactic treatment with oral antibiotics should also be considered.

The results from the previous study were supported in another retrospective study that measured infection rates after injections with Aquamid in 657 subjects from one site. ${ }^{20}$ Subjects had facial injections with Aquamid from 2001 and 2011. Prior to 2007, prophylactic antibiotics were not administered before treatment. However, beginning in September 2007 prophylactic single oral doses of azithromycin and moxifloxacin were introduced. In this study, 496 subjects were injected prior to 2007 without prophylactic antibiotic therapy, and 161 subjects received azithromycin and moxifloxacin prior to injections starting in September 2007. Prophylactic treatment with antibiotics significantly reduced the incidence of inflammation and infections from $7 \%$ to $2 \%$. Even though the rates of infection after injection with Aquamid were relatively low, the use of prophylactic antibiotic treatment further lowered the incidence of infections and inflammation. Based on the author's experience, they 
recommend the administration of prophylactic antibiotics to patients who have had facial injections with Aquamid to prevent infection and risk of biofilm formation from contamination with normal skin flora.

\section{Summary}

Aquamid is currently approved in several countries in Europe, Asia, the Middle East, and Latin America but is not available in the US although a 24-month efficacy and safety clinical trial was performed there. Many studies have supported the usage of Aquamid for the treatment of various rhytides, facial contouring, and correction of HIV lipoatrophy. For the past decade, Aquamid has gained popularity as an injectable filler. Similar to other facial fillers, there have been reported cases of inflammation, nodule and granuloma formation, and delayed hypersensitivity reactions. In some instances, surgical extraction of the polyacrylamide product was necessary to correct the adverse event of nodule formation. Careful attention to injection technique and sterile precautions are necessary to minimize unwanted reactions. In addition, there have been recent recommendations for the usage of prophylactic antibiotics to minimize complications from bacterial injections and biofilm formation when injecting Aquamid..$^{19,20}$

\section{Disclosure}

The author reports no conflicts of interest in this work.

\section{References}

1. Christensen LH, Breiting VB, Aasted A, Jørgensen A, Kebuladze I. Long-term effects of polyacrylamide hydrogel on human breast tissue. Plast Reconstr Surg. 2003;111(6):1883-1890.

2. Fernández-Cossío S, Castaño-Oreja MT. Biocompatibility of two novel dermal fillers: histological evaluation of implants of a hyaluronic acid filler and a polyacrylamide filler. Plast Reconstr Surg. 2006;117(6): 1789-1796.

3. Lemperle G, Morhenn V, Charrier U. Human histology and persistence of various injectable filler substances for soft tissue augmentation. Aesthetic Plast Surg. 2003;27(5):354-366.

4. de Cássia Novaes W, Berg A. Experiences with a new nonbiodegradable hydrogel (Aquamid): a pilot study. Aesthetic Plast Surg. 2003;27(5): 376-380.

5. von Buelow S, von Heimburg D, Pallua N. Efficacy and safety of polyacrylamide hydrogel for facial soft-tissue augmentation. Plast Reconstr Surg. 2005;116(4):1137-1146.
6. von Buelow S, Pallua N. Efficacy and safety of polyacrylamide hydrogel for facial soft-tissue augmentation in a 2-year follow-up: a prospective multicenter study for evaluation of safety and aesthetic results in 101 patients. Plast Reconstr Surg. 2006;118(Suppl 3):85S-91S.

7. Pallua N, Wolter TP. A 5 -year assessment of safety and aesthetic results after facial soft-tissue augmentation with polyacrylamide hydrogel (Aquamid): a prospective multicenter study of 251 patients. Plast Reconstr Surg. 2010;125(6):1797-1804.

8. Wolters M, Lampe H. Prospective multicenter study for evaluation of safety, efficacy, and esthetic results of cross-linked polyacrylamide hydrogel in 81 patients. Dermatol Surg. 2009;35(Suppl 1):338-343.

9. Lari A. Augmentation of the malar area with polyacrylamide hydrogel: experience with more than 1300 patients. Aesthet Surg J. 2008;28(2):131-138.

10. Lafaurie M, Dolivo M, Girard PM, et al. ANRS 132 SMILE study group. Polylactic acid vs polyacrylamide hydrogel for treatment of facial lipoatrophy: a randomized controlled trial [Agence Nationale de Recherches sur le SIDA et les Hépatites Virales (ANRS) 132 SMILE]. HIV Med. 2013;14(7):410-420.

11. Rauso R, Freda N, Parlato V, Gherardini G, Amore R, Tartaro G. Polyacrylamide gel injection for treatment of human immunodeficiency virus-associated facial lipoatrophy: 18 months follow-up. Dermatol Surg. 2011;37(11):1584-1589.

12. Mole B, Gillaizeau F, Carbonnel E, et al. Polyacrylamide hydrogel injection in the management of human immunodeficiency virus-related facial lipoatrophy: results of the LIPOPHILL open-label study. AIDS Res Hum Retroviruses. 2012;28(3):251-258.

13. Rauso R, Curinga G, Santillo V, Corvo G, Tartaro G. Comparison between lipofilling and a nonabsorbable filler for facial wasting rehabilitation in HIV-positive patients. J Craniofac Surg. 2011;22(5):1684-1688.

14. Rauso R, Gherardini G, Parlato V, Amore R, Tartaro G. Polyacrylamide gel for facial wasting rehabilitation: how many milliliters per session? Aesthetic Plast Surg. 2012;36(1):174-179.

15. Manafi A, Emami AH, Pooli AH, Habibi M, Saidian L. Unacceptable results with an accepted soft tissue filler: polyacrylamide hydrogel. Aesthetic Plast Surg. 2010;34(4):413-422.

16. Kalantar-Hormozi A, Mozafari N, Rasti M. Adverse effects after use of polyacrylamide gel as a facial soft tissue filler. Aesthet Surg J. 2008;28(2):139-142.

17. Alijotas-Reig J, Garcia-Gimenez V, Miró-Mur F, Vilardell-Tarrés M. Delayed immune-mediated adverse effects related to polyacrylamide dermal fillers: clinical findings, management, and follow-up. Dermatol Surg. 2009;35(Suppl 1):360-366.

18. Shen H, Lv Y, Xu JH, et al. Complications after polyacrylamide hydrogel injection for facial soft-tissue augmentation in China: twenty-four cases and their surgical management. Plast Reconstr Surg. 2012; 130(2):340e-348e.

19. Christensen L, Breiting V, Bjarnsholt T, et al. Bacterial infection as a likely cause of adverse reactions to polyacrylamide hydrogel fillers in cosmetic surgery. Clin Infect Dis. 2013;56(10):1438-1444.

20. Nygart JF, Nygart VA, Borggren M, Tvede M. Effect of prophylactic antibiotics on polyacrylamide gel safety in facial augmentation. J Drugs Dermatol. 2014;13(5):571-573.
Clinical, Cosmetic and Investigational Dermatology

\section{Publish your work in this journal}

Clinical, Cosmetic and Investigational Dermatology is an international, peer-reviewed, open access, online journal that focuses on the latest clinical and experimental research in all aspects of skin disease and cosmetic interventions. All areas of dermatology will be covered; contributions will be welcomed from all clinicians and

\section{Dovepress}

basic science researchers globally. This journal is indexed on CAS The manuscript management system is completely online and includes a very quick and fair peer-review system, which is all easy to use. Visit http://www.dovepress.com/testimonials.php to read real quotes from published authors. 